\title{
Reducing the Uncertainty: Gating of Peripheral Inputs by Zona Incerta
}

\author{
Jason C. Trageser and Asaf Keller \\ Program in Neuroscience and Department of Anatomy and Neurobiology, University of Maryland School of Medicine, Baltimore, Maryland 21201
}

\begin{abstract}
Sensory inputs are relayed to the neocortex by "first-order" thalamic nuclei, the responses of which are determined by ascending inputs from peripheral receptors. In contrast, "higher-order" thalamic nuclei respond poorly to peripheral inputs, and their responses are thought to be determined by descending cortical inputs. We tested this hypothesis by recording from neurons in the higher-order somatosensory posterior medial (POm) nucleus of narcotized rats. As reported previously, POm neurons responded to whisker stimuli with long-latency (median, $27 \mathrm{msec}$ ) and low-magnitude responses, consistent with cortically driven responses. However, when we suppressed inhibitory inputs from the subthalamic nucleus zona incerta (ZI), POm responses were of significantly higher magnitude and shorter latency, with many POm neurons responding at latencies consistent with ascending driving inputs from trigeminal nuclei. Our data suggest that POm comprises two neuronal populations: one population is driven by both peripheral and cortical inputs, and the second population responds only to cortical inputs. These findings demonstrate that ZI gates peripheral inputs to POm, enabling it to function both as a first-order and higher-order nucleus. Because ZI innervates all higher-order nuclei, this gating mechanism may exert similar regulation of thalamic processing in other sensory systems.
\end{abstract}

Key words: posterior medial nucleus; thalamus; first-order nucleus; higher-order nucleus; thalamocortical; corticothalamic

\section{Introduction}

Sensory inputs are relayed to the neocortex by thalamic nuclei termed "first-order" relays [e.g., the lateral geniculate nucleus in the visual system and the ventral posterior medial (VPM) nucleus in the somatosensory system] (Jones, 1985). In addition, a number of thalamic nuclei, such as the pulvinar of the visual system, are hypothesized to be involved in relaying information from one cortical area to another and have been termed "higher-order" relays (Sherman and Guillery, 2001).

First-order nuclei contain neurons with receptive field properties that are determined by ascending inputs (e.g., from the retina or brainstem) that drive these cells to firing threshold. Higher-order nuclei contain neurons with drivers that are descending cortical inputs and receptive fields that are determined by these descending projections. Morphologically, driver afferents, whether cortical or subcortical, are characterized by thick and highly branched (type II) axons with large terminals (Guillery, 1966; Jones, 1983).

In the somatosensory system, the posterior medial thalamic nucleus (POm) is thought to function as a higher-order relay that is driven by descending cortical inputs. POm receives dense innervation from corticothalamic axons, the morphological criteria of which suggest that they are driver afferents (Hoogland et al.,

Received Aug. 5, 2004; revised Sept. 3, 2004; accepted Sept. 4, 2004.

This work was supported by United States Public Health Service-National Institute of Neurological Disorders and Stroke Grants NS-31078 and NS-35360 to A.K. and Grant 1F31NS046123 to J.C.T.

Correspondence should be addressed to Dr. Asaf Keller, Department of Anatomy and Neurobiology, University of Maryland School of Medicine, 20 Penn Street, Baltimore, MD 21201. E-mail: akeller@umaryland.edu. DOI:10.1523/JNEUROSCI.3218-04.2004

Copyright $\odot 2004$ Society for Neuroscience $\quad$ 0270-6474/04/248911-05\$15.00/0
1991; Deschênes et al., 1994). Indeed, Diamond et al. (1992a) showed that POm neurons respond to whisker stimuli at latencies that are significantly longer than those of VPM cells (mean, 19 vs $7 \mathrm{msec}$ ) and that these POm responses are abolished after cortical inactivation (Diamond et al., 1992b). These and other findings (Killackey and Sherman, 2003) support the designation of POm as a higher-order nucleus.

However, there are anatomical and physiological data suggesting that POm receives driving brainstem inputs. Anatomically, POm receives inputs from brainstem afferents that fulfill the morphological criteria of driver inputs (Veinante et al., 2000). Physiologically, Chiaia et al. (1991) showed that electrically stimulating the trigeminal nucleus principalis or the subnucleus interpolaris in the brainstem generates action potentials with similar onset latencies in the VPM and POm, suggesting that both nuclei respond in a similar manner to brainstem inputs. Furthermore, Ahissar et al. (2000) reported that some POm neurons respond to whisker deflections at short latencies (7-8 msec). Together, these data indicate that POm can be driven by brainstem inputs and may therefore also function as a first-order nucleus.

Why do subcortical inputs rarely result in short-latency whisker-evoked responses in POm neurons? Here, we tested the hypothesis that the subthalamic nucleus zona incerta (ZI) suppresses subcortical inputs to POm. [We are indebted to Prof. M. Deschênes (Laval University, Quebec City, Canada) for fostering this idea.] The ZI provides dense GABAergic inputs to POm neurons (Power et al., 1999; Bartho et al., 2002), suggesting that it exerts potent inhibition on $\mathrm{POm}$ neurons preventing it from reliably responding to subcortical activation.

We show that suppressing ZI-mediated inhibition reveals robust short-latency whisker-evoked responses in POm neurons. 
Our findings suggest that POm functions both as a first-order relay (driven by brainstem inputs) and a higher-order relay (driven by cortical inputs). We propose a model in which ZI (Greek for "zone of uncertainty") selectively gates these inputs, depending on the behavioral state of the animal.

\section{Materials and Methods}

Surgical procedures. Nineteen female Sprague Dawley rats weighing 250-350 gm were used in this study. All procedures strictly adhered to institutional and federal guidelines. Under halothane anesthesia (3\%) and infusion of local anesthetics at surgical sites, a craniotomy was performed over the right POm thalamus and ZI. The dura was removed, and agarose $(1.4 \%$ in buffered saline) was poured over the craniotomy to prevent drying. A venous catheter was inserted in the jugular vein for drug delivery, and a second catheter was placed in the femoral artery for monitoring blood pressure and heart rate. After the surgical procedures, administration of halothane was discontinued, and the rats were infused intravenously with fentanyl (10 $\mu \mathrm{g} / \mathrm{kg} / \mathrm{hr}$ ) for the rest of the experiment. The rats were then immobilized with pancuronium bromide $(1.5 \mathrm{mg} / \mathrm{kg} / \mathrm{hr})$ and artificially respired with a positive pressure respirator at 90 breaths $/ \mathrm{min}$. Blood pressure, heart rate, and electroencephalographs were monitored throughout the experiment to ensure that the animal was in no pain or distress. Body temperature was maintained at $37^{\circ} \mathrm{C}$ with a servo-controlled heating blanket.

Recording. Extracellular unit recordings were obtained with quartzinsulated platinum electrodes $(2-4 \mathrm{M} \Omega$ ). Electrodes were advanced to the POm thalamus in the right hemisphere based on stereotaxic coordinates [anteroposterior (AP), 3.2; mediolateral (ML), 2.6]. Animals were maintained within the stereotaxic frame throughout the recordings. Whiskers on the contralateral face were stimulated continually during electrode penetrations to detect units with low or no spontaneous activity. Waveforms recorded from well isolated units were digitized through a data acquisition system (Plexon, Dallas, TX) at $40 \mathrm{kHz}$. Units were isolated off-line with Offline Sorter (Plexon), and auto-correlograms were generated with Neuroexplorer (Littleton, MA) software to confirm that recordings were obtained from single units.

At the end of the experiment, recording sites were marked with electrolytic lesions ( $5 \mu \mathrm{A}$ for $10 \mathrm{sec}$ ). The animals were then deeply anesthetized with sodium pentobarbital $(60 \mathrm{mg} / \mathrm{kg})$ and perfused transcardially with buffered saline, followed by $4 \%$ buffered paraformaldehyde. Tissue sections were Nissl stained, and recording sites were identified in coronal sections.

ZI inactivation and histology. In six of the 19 rats, before recording POm neurons, we identified the whisker representation in the ZI by advancing a recording electrode into the nucleus following stereotaxic coordinates (AP, 3.5; ML, 2.8). We continuously stimulated the contralateral whiskers until well isolated units responding to the stimulation were identified. We then replaced the recording electrode with a blunt tungsten metal electrode $(0.5 \mathrm{M} \Omega ; 200 \mu \mathrm{m}$ tip diameter $)$ and created an electrolytic lesion by passing $2.5 \mathrm{~mA}$ of negative current for $4 \mathrm{sec}$. Verification of lesion sites was performed as described above. Only animals with clear lesions within the ZI were included in the analyses. POm recordings were obtained within 30 min of creating lesions in the $\mathrm{ZI}$, and the recording session lasted up to $6 \mathrm{hr}$. No significant differences were observed in any of the parameters over the course of an experiment.

Whisker stimulation. After isolation of whisker-sensitive neurons in POm, whiskers were stimulated by air puffs delivered through a tube $(0.5$ $\mathrm{mm}$ diameter) by a computer-controlled Picospritzer. Air puffs were
B

$\mathrm{C}$

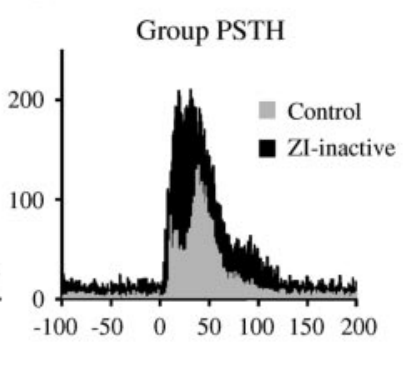

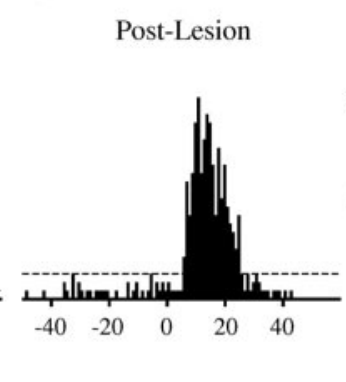
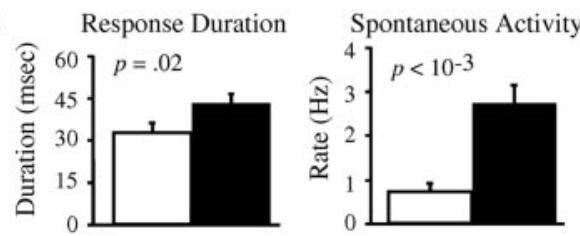

$$
{ }_{0}
$$

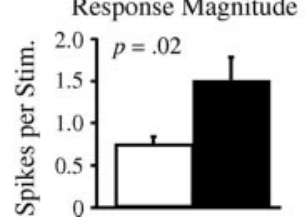

Figure 1. Effects of Zl inactivation on POm response properties. $A, B$, PSTHs constructed from responses of POm neurons to a 50 control neurons and 42 neurons recorded after $Z 1$ inactivation. $D$, Response onset latency, response magnitude, response duration, and spontaneous activity rates were all significantly affected by Zl inactivation. Bar graphs depict mean \pm SEM. $p$ values were computed with Mann-Whitney $U$ test.

delivered at $1 \mathrm{~Hz}$ with a pressure of $60 \mathrm{psi}$, resulting in whisker deflections of $\sim 30^{\circ}$. Whiskers were deflected in their preferred direction (i.e., the direction that elicited the shortest latency, highest magnitude response). At least four whiskers were deflected simultaneously to evoke responses in POm neurons.

Data analysis. Time stamps of well isolated units and of stimulus triggers were exported to Matlab (MathWorks, Natick, MA) for analyses using custom-written algorithms. Peristimulus time histograms (PSTHs; $1 \mathrm{msec}$ bins) were constructed from these time stamps. Significant stimulus-evoked responses were defined as PSTH bins with a response magnitude that significantly exceeded ( $99 \%$ confidence interval) spontaneous activity levels, computed from a $200 \mathrm{msec}$ period preceding the stimuli.

We defined response onset as the first two consecutive bins (poststimulus) displaying significant responses (defined as above), and response offset was defined as two consecutive bins in which response magnitude fell below the $99 \%$ confidence interval. We defined response magnitude as the total number of spikes per stimulus occurring between response onset and offset.

Statistical analyses were performed in SPSS (SPSS, Chicago, IL). Between-group statistical comparisons were assessed with the nonparametric Mann-Whitney $U$ test. Data are presented as means \pm SEM.

\section{Results}

\section{POm responses to whisker stimuli}

In fentanyl-narcotized rats, we characterized responses of well isolated POm neurons to whisker stimuli, evoked by deflecting whiskers with air puffs (see Materials and Methods). Typical response properties of a representative POm neuron are depicted in the PSTH in Figure $1 \mathrm{~A}$. POm neurons responded poorly to whisker stimuli, requiring large amplitude deflections of multiple whiskers to evoke spikes. Response magnitudes were variable [coefficient of variation (CV), 0.82] and low (median, 0.65 spikes/stimulus; mean \pm SEM, $0.74 \pm 0.096$ spikes/stimulus) (Fig. $1 D)$. Also variable $(\mathrm{CV}, \geq 0.51)$ were response duration (median, $28.0 \mathrm{msec} ; 32.8 \pm 3.2 \mathrm{msec}$ ) and spontaneous firing rates (median, $0.2 \mathrm{~Hz} ; 0.7 \pm 0.19 \mathrm{~Hz}$ ). As a group (Fig. $1 \mathrm{D})$, POm 


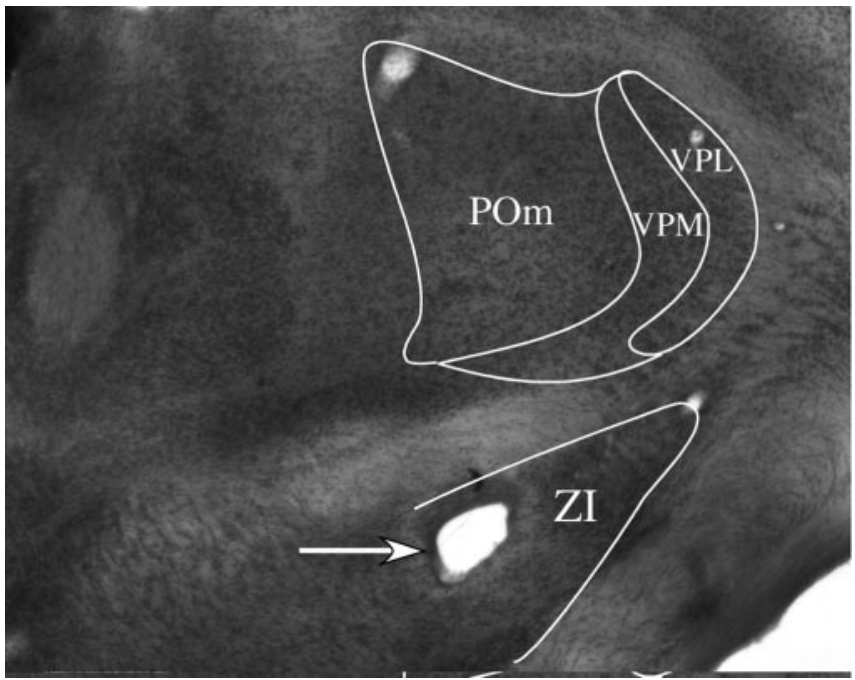

Figure 2. Coronal section through the thalamus illustrating the anatomical relationship between the thalamic nuclei POm, VPM, ventral posterior lateral (VPL), and the subthalamic nucleus Zl; note the lesion (arrowhead) localized to the dorsal/ventral subregion of the ZI.

neurons responded at variable latencies, ranging from 6 to 66 msec (median, $27.0 \mathrm{msec} ; 27.4 \pm 2.2 \mathrm{msec}$ ).

These results are consistent with previous reports on POm neurons recorded under urethane (Chiaia et al., 1991; Diamond et al., 1992a; Sosnik et al., 2001), demonstrating that under anesthesia, as well as under fentanyl analgesia (present study), these neurons respond poorly and inconsistently to whisker stimuli.

\section{$\mathrm{ZI}$ regulates $\mathrm{POm}$ responses}

The weak and labile responses of POm neurons are surprising in light of the fact that POm receives direct, short-latency inputs from trigeminal nuclei (see Introduction). We reasoned, therefore, that $\mathrm{POm}$ responses might be suppressed by GABAergic inhibition. The source of GABAergic inhibition to POm is likely to arise from extrinsic sources, because $\mathrm{POm}$ in the rat is reported to contain few, if any, GABAergic neurons (Barbaresi et al., 1986; Harris and Hendrickson, 1987). These extrinsic GABAergic sources include the thalamic reticular nucleus (TRN) (Pinault and Deschênes, 1998), pretectal and mesencephalic nuclei, and the ZI (Power et al., 1999; Acsady et al., 2003). We chose to focus on the role of GABAergic inputs from the ZI for the following reasons. Unlike the other nuclei, ZI neurons respond at short latency (4 msec) to whisker stimuli (Nicolelis et al., 1992; our unpublished observations), suggesting that they may mediate feed-forward inhibition of POm neurons. Furthermore, unlike TRN axons, which terminate on distal dendrites of thalamocor $\backslash$ tical projection neurons (Peschanski et al., 1983; Wang et al., 2001), ZI axons synapse on the perisomatic regions of these cells (Bartho et al., 2002), suggesting that they exert potent tonic inhibition of POm neurons.

To test the role of the $\mathrm{ZI}$ in regulating $\mathrm{POm}$ responses, we inactivated ZI by placing electrolytic lesions in the physiologically identified whisker representation of the ZI (Fig. 2) (see Materials and Methods). The lesions were $300-500 \mu \mathrm{m}$ in diameter and included the dorsal/ventral subregions of the ZI, which contain somatotopic representations of the whiskers (Nicolelis et al., 1992). We compared data obtained from neurons recorded from the control group (rats with intact ZI) with data from neurons recorded from the ZI inactivated group (rats receiving ZI lesions before POm recordings). Figure 1 depicts representative PSTHs recorded from two different neurons. The control neuron (Fig. $1 A$ ) had no spontaneous activity and responded to whisker stimuli at a latency of $26 \mathrm{msec}$. In contrast, the cell recorded after the $\mathrm{ZI}$ lesion (Fig. $1 \mathrm{~B}$ ) fired spontaneously at $9 \mathrm{~Hz}$ and responded to whisker deflections at $6 \mathrm{msec}$. Similar results were obtained from 80 additional neurons (39 control and 41 postlesion) (Fig. 1D). POm neurons from rats having lesions responded significantly ( $p=0.03$; Mann-Whitney $U$ test) earlier (median, $16.0 \mathrm{msec}$; $21.2 \pm 2.4 \mathrm{msec}$ ) than did neurons recorded from controls (median, $27.0 \mathrm{msec} ; 27.4 \pm 2.2 \mathrm{msec}$ ). Inactivating ZI also affected the kinetics of POm responses, as depicted in the group PSTHs (Fig. 1C), constructed by pooling data from 40 control neurons and 42 neurons recorded from the ZI inactivated group. Both response magnitude (median, 0.84 vs 0.65 spikes/stimulus) and duration ( 40.0 vs $28.0 \mathrm{msec}$ ) were significantly ( $p=0.02)$ increased, and spontaneous firing rates were significantly $(p<$ $10^{-3}$ ) higher after ZI inactivation (median, $1.7 \mathrm{~Hz}$ ) compared with control (median, $0.2 \mathrm{~Hz}$ ) (Fig. 1D). These findings demonstrate that inactivation of the ZI reveals in POm neurons robust, short-latency responses to whisker deflections.

\section{POm contains two neuronal populations}

The PSTH constructed by pooling data from control neurons (Fig. 1C) has a bimodal distribution, suggesting that some control neurons respond to whisker deflections at relatively short latencies. (This bimodal distribution in the group data does not represent biphasic response kinetics in individual neurons because PSTHs computed for individual neurons were always monophasic.) To quantify the distribution of onset latencies in both control and postlesion neurons, we constructed cumulative probability plots (Fig. $3 A$ ): these demonstrate a leftward shift in the distribution of latencies $\leq 30 \mathrm{msec}$ and an overlapping distribution of latencies $>30 \mathrm{msec}$. This suggests either that we failed to suppress ZI inputs to some POm neurons or that a subset of these neurons fails to respond to whisker inputs at short latency, even when the ZI is suppressed.

To distinguish between these possibilities, we compared the response kinetics of control $(n=10)$ and postlesion $(n=13)$ neurons that responded at latencies $>30 \mathrm{msec}$ (Fig. $3 B$ ). In this group, ZI lesions had no significant effect on onset latency ( $p=$ $0.28)$ or response magnitude $(p=0.38)$ and duration $(p=$ $0.61)$. However, spontaneous activity rates were significantly $(p=0.002)$ higher (median, $2.4 \mathrm{~Hz} ; 2.5 \pm 0.38 \mathrm{~Hz})$ after ZI lesions compared with control (median, $0.1 \mathrm{~Hz} ; 0.6 \pm 0.47 \mathrm{~Hz}$ ). The increase in spontaneous activity suggests that ZI lesions were effective in relieving tonic inhibition from this group of POm neurons. Thus, the fact that ZI lesions did not affect response latency or kinetics in this group is not likely attributable to incomplete suppression of their ZI inputs. Rather, this group of POm neurons may represent a distinct population of cells that receive no direct trigeminal inputs.

In contrast, ZI lesions had significant effects on the group of POm neurons that respond at $\leq 30 \mathrm{msec}(n=30$ control; $n=29$ postlesion). The lesions resulted in a leftward shift in onset latency, demonstrating that ZI lesions significantly $\left(p<10^{-3}\right)$ increased the proportion of neurons responding to whisker stimuli at short latency (median, $12.0 \mathrm{msec} ; 12.3 \pm 1.3 \mathrm{msec}$ vs median, $24.0 \mathrm{msec} ; 21.0 \pm 1.5 \mathrm{msec}$ ). In this group of neurons, ZI lesions resulted also in significant increases in spontaneous activity ( $p=0.01$; median, $1.4 \mathrm{~Hz} ; 2.6 \pm 0.54 \mathrm{~Hz}$ vs median, $0.4 \mathrm{~Hz} ; 0.7 \pm$ $0.20 \mathrm{~Hz}$ ), response magnitude ( $p=0.008$; median, 1.2 spikes/stimulus; $1.9 \pm 0.36$ spikes/stimulus vs median, 0.79 spikes/stimulus; $0.85 \pm 0.11$ spikes/stimulus), and response duration $(p=0.02$; 
median, $40.0 \mathrm{msec} ; 42.0 \pm 3.9 \mathrm{msec}$ vs median, $24.0 \mathrm{msec} ; 30.0 \pm 3.2 \mathrm{msec}$ ) (Fig. 3C).

\section{Discussion}

\section{Methodological considerations}

We chose to inactivate ZI by producing electrolytic lesions because this structure is large and wraps around the ventral aspect of the dorsal thalamus, rendering it difficult to inactivate pharmacologically. Although electrolytic lesions may inadvertently affect axons of passage, this is not likely to be a concern because there are no known GABAergic axons coursing through the ZI on their way to POm.

The majority $(>90 \%)$ of incertothalamic afferents are GABAergic (Bartho et al., 2002). Although there are reports that some ZI efferents are glutamatergic (Kolmac and Mitrofanis, 1998), others have argued that this result may represent a labeling artifact (Bartho et al., 2002). Our finding that ZI lesions reveal short-latency POm responses is inconsistent with a dominant glutamatergic incerto-thalamic pathway (or excitatory fibers-of-passage coursing through the ZI). If such glutamatergic pathways existed, we would expect ZI lesions to suppress whisker-evoked responses rather than reveal the short-latency responses we observed. We therefore conclude that our lesions of the ZI selectively inactivated incerto-thalamic GABAergic inputs.

\section{$\mathrm{ZI}$ regulates $\mathrm{POm}$ responses}

Our findings demonstrate that inactivating the ZI has a profound effect on POm responses. Median spontaneous activity rates were eightfold higher in neurons recorded after lesions compared with controls, suggesting that the ZI exerts tonic inhibition on POm neurons. This conclusion is supported by the fact that GABAergic afferents from ZI synapse on perisomatic regions of POm neurons (Bartho et al., 2002), where they are likely to exert potent postsynaptic inhibition, and by the fact that ZI neurons fire spontaneously (Eaton and Moss, 1989; Koyama et al., 2003).

Inactivating the ZI also affected the responses of POm neurons to whisker stimuli. Response latencies in rats with lesions were significantly shorter than in controls, and both the magnitude and duration of the responses were significantly increased. Indeed, after ZI lesions, response latencies of many POm neurons were similar to those of VPM cells ( $8 \mathrm{msec}$ ) (Ito, 1988; Hartings and Simons, 1998; Ahissar et al., 2000; Sosnik et al., 2001). Whereas in the control group, only $12 \%$ of POm neurons responded to whisker stimuli at these latencies, in the ZI inactivated group, 33\% responded at $\leq 8 \mathrm{msec}$ (Fig. $3 A$ ). These findings are consistent with the hypothesis that $\mathrm{POm}$ receives driving inputs from ascending trigeminal afferents and that the efficacy of these inputs is regulated by inhibitory inputs from the ZI.

Not all POm neurons were equally affected by inactivating the ZI: in $\sim 30 \%$ of the cells recorded from the ZI inactivated group, the lesions did not affect the latency or kinetics of whisker-evoked responses (Fig. 3). The fact that inactivating the $\mathrm{ZI}$ increased the spontaneous firing in this subpopulation of neurons suggests that the lesions were effective in suppressing ZI inputs to these neurons. Together, these findings suggest that POm contains two neuronal populations, one that receives driving brainstem inputs
B

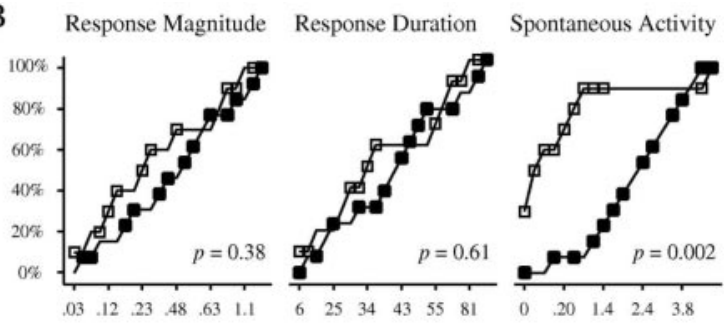

C
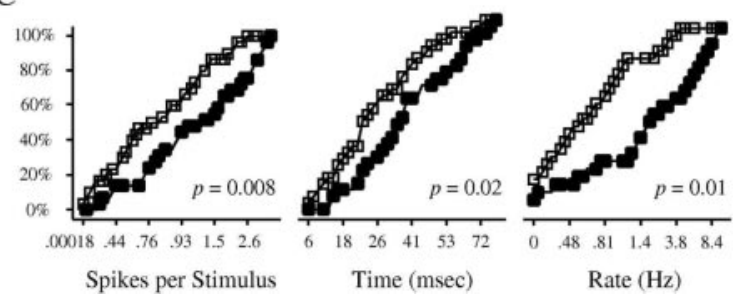

Figure 3. POm comprises two neuronal populations. $A$, Cumulative probability plots showing the distribution of response onset latencies of $\mathrm{PO} \mathrm{m}$ neurons recorded from control rats versus neurons recorded after $\mathrm{Zl}$ inactivation. Zl inactivation results in msec. In this group, Zl inactivation resulted in significant increases in spontaneous firing rates but had no effect on response magnitude or duration. C, Cumulative probability plots constructed for the group of P0m neurons that responded at $\leq 30 \mathrm{msec}$ All metrics were significantly affected by Zl inactivation in this group.

that are modulated by inhibitory inputs from the ZI and one that receives few or weak inputs from brainstem afferents and is primarily driven by descending cortical inputs.

\section{POm as both a first- and higher-order nucleus}

Our findings suggest that the dichotomy between first-order thalamic nuclei (driven by ascending sensory afferents) and higherorder nuclei (driven by descending cortical inputs) may not apply to the POm. Although POm has been conclusively shown to function as a higher-order nucleus (see Introduction), our findings demonstrate that it functions also as a first-order nucleus with response properties that are shaped by ascending trigeminal inputs.

To our knowledge, our findings provide the first support for the conjecture originally proposed by Sherman et al. (2001) that "it is possible that several thalamic nuclei may represent a mixture of the two types of circuit, having some afferents from lower centers contributing to first-order circuits and others coming from the cortex and contributing to higher-order circuits ... The possibility that two such pathways might interact in the thalamus remains completely unexplored.”

\section{ZI affects all higher-order thalamic nuclei}

GABAergic incerto-thalamic afferents target all higher-order thalamic nuclei (but do not project to first-order nuclei) (Power et al., 1999; Bartho et al., 2002). It is therefore likely that the regulation of POm responses by these afferents applies also to higherorder thalamic nuclei of other sensory systems. For example, the pulvinar is classified as a higher-order thalamic nucleus of the visual system (Sherman and Guillery, 2001). However, retinal axons innervate the pulvinar (Grieve et al., 2000), suggesting that ascending inputs may drive these neurons. Furthermore, retinal axons innervate the $\mathrm{ZI}$, which in turn projects to the pulvinar (Power et al., 1999, 2001). This anatomical connectivity is analogous to the one in the somatosensory system (see Introduction). It is therefore possible that the pulvinar, like POm, may function 
both as a first-order and a higher-order nucleus and that these functions are regulated by inhibitory inputs from the ZI. Thus, the dichotomy between first-order and higher-order nuclei of all sensory systems may need to be revised to include the dual functions of thalamic nuclei.

\section{References}

Acsady L, Bokor H, Slezia A (2003) Multiple extrareticular source of GABAergic afferents to higher order thalamic nuclei. Soc Neurosci Abstr 29:60.69.

Ahissar E, Sosnik R, Haidarliu S (2000) Transformation from temporal to rate coding in a somatosensory thalamocortical pathway. Nature 406:302-306.

Barbaresi P, Spreafico R, Frassoni C, Rustioni A (1986) GABAergic neurons are present in the dorsal column nuclei but not in the ventroposterior complex of rats. Brain Res 382:305-326.

Bartho P, Freund TF, Acsady L (2002) Selective GABAergic innervation of thalamic nuclei from zona incerta. Eur J Neurosci 16:999-1014.

Chiaia NL, Rhoades RW, Fish SE, Killackey HP (1991) Thalamic processing of vibrissal information in the rat. II. Morphological and functional properties of medial ventral posterior nucleus and posterior nucleus neurons. J Comp Neurol 314:217-236.

Deschênes M, Bourassa J, Pinault D (1994) Corticothalamic projections from layer $\mathrm{V}$ cells in rat are collaterals of long-range corticofugal axons. Brain Res 664:215-219.

Diamond ME, Armstrong-James M, Ebner FF (1992a) Somatic sensory responses in the rostral sector of the posterior group (POm) and in the ventral posterior medial nucleus (VPM) of the rat thalamus. J Comp Neurol 318:462-476.

Diamond ME, Armstrong-James M, Budway MJ, Ebner FF (1992b) Somatic sensory responses in the rostral sector of the posterior group (POm) and in the ventral posterior medial nucleus (VPM) of the rat thalamus: dependence on the barrel field cortex. J Comp Neurol 319:66-84.

Eaton MJ, Moss RL (1989) Electrophysiology and pharmacology of neurons of the medial zona incerta: an in vitro slice study. Brain Res 502:117-126.

Grieve KL, Acuna C, Cudeiro J (2000) The primate pulvinar nuclei: vision and action. Trends Neurosci 23:35-39.

Guillery RW (1966) A study of Golgi preparations from the dorsal lateral geniculate nucleus of the adult cat. J Comp Neurol 128:21-50.

Harris RM, Hendrickson AE (1987) Local circuit neurons in the rat ventrobasal thalamus-a GABA immunocytochemical study. Neuroscience 21:229-236.

Hartings JA, Simons DJ (1998) Thalamic relay of afferent responses to 1- to 12-Hz whisker stimulation in the rat. J Neurophysiol 80:1016-1019.
Hoogland PV, Wouterlood FG, Welker E, Van der Loos H (1991) Ultrastructure of giant and small thalamic terminals of cortical origin: a study of the projections from the barrel cortex in mice using Phaseolus vulgaris leuco-agglutinin (PHA-L). Exp Brain Res 87:159-172.

Ito M (1988) Response properties and topography of vibrissa-sensitive VPM neurons in the rat. J Neurophysiol 60:1181-1197.

Jones EG (1983) Distribution patterns of individual medial lemniscal axons in the ventrobasal complex of the monkey thalamus. J Comp Neurol 215:1-16.

Jones EG (1985) The thalamus. New York: Plenum.

Killackey HP, Sherman SM (2003) Corticothalamic projections from the rat primary somatosensory cortex. J Neurosci 23:7381-7384.

Kolmac CI, Mitrofanis J (1998) Distribution of various neurochemicals within the zona incerta: an immunocytochemical and histochemical study. Anat Embryol 199:265-280.

Koyama Y, Takahashi K, Kodama T, Kayama Y (2003) State-dependent activity of neurons in the perifornical hypothalamic area during sleep and waking. Neuroscience 119:1209-1219.

Nicolelis MA, Chapin JK, Lin RC (1992) Somatotopic maps within the zona incerta relay parallel GABAergic somatosensory pathways to the neocortex, superior colliculus, and brainstem. Brain Res 577:134-141.

Peschanski M, Ralston HJ, Roudier F (1983) Reticularis thalami afferents to the ventrobasal complex of the rat thalamus: an electron microscope study. Brain Res 270:325-329.

Pinault D, Deschênes M (1998) Projection and innervation patterns of individual thalamic reticular axons in the thalamus of the adult rat: a threedimensional, graphic, and morphometric analysis. J Comp Neurol 391:180-203.

Power BD, Kolmac CI, Mitrofanis J (1999) Evidence for a large projection from the zona incerta to the dorsal thalamus. J Comp Neurol 404:554-565.

Power BD, Leamey CA, Mitrofanis J (2001) Evidence for a visual subsector within the zona incerta. Vis Neurosci 18:179-186.

Sherman SM, Guillery RW (2001) Exploring the thalamus. San Diego: Academic.

Sosnik R, Haidarliu S, Ahissar E (2001) Temporal frequency of whisker movement. I. Representations in brain stem and thalamus. J Neurophysiol 86:339-353.

Veinante P, Jacquin MF, Deschênes M (2000) Thalamic projections from the whisker-sensitive regions of the spinal trigeminal complex in the rat. J Comp Neurol 420:233-243.

Wang ST, Bickford ME, Van Horn SC, Erisir A, Godwin DW, Sherman SM (2001) Synaptic targets of thalamic reticular nucleus terminals in the visual thalamus of the cat. J Comp Neurol 440:321-341. 